\title{
Spatial Turnover Overrides Temporal Turnover In Two Plant-Pollinator Communities
}

\author{
Ainhoa Magrach ( $\square$ ainhoamagrach@hotmail.com ) \\ Basque Center for Climate Change https://orcid.org/0000-0003-2155-7556 \\ Maddi Artamendi \\ Universidad del País Vasco: Universidad del Pais Vasco \\ Paula Dominguez-Lapido \\ Basque Center for Climate Change \\ Clara Parejo \\ Basque Center for Climate Change \\ Encarnacion Rubio \\ Basque Center for Climate Change
}

\section{Research Article}

Keywords:

Posted Date: February 23rd, 2022

DOI: https://doi.org/10.21203/rs.3.rs-1326633/v1

License: (c) (i) This work is licensed under a Creative Commons Attribution 4.0 International License.

Read Full License 


\section{Abstract}

In recent years, an extended body of literature has focused on the importance of temporal dynamics in shaping the structure of plant and pollinator communities and their interactions. This improvement from a previously static perspective to community analysis has allowed us to understand many of the ecological processes that shape these communities. However, a focus on temporal dynamics has led to a decreased attention to the spatial dynamics. Here, we use a dataset collected across two contrasting habitat types in N and SW Spain, mountain grasslands and the understory of sparse pine forests. We collected data across five sites in each area, and weekly throughout the full flowering season at each area for two consecutive years. Our results show that spatial differences in species composition and species individual roles led to significant changes in interaction composition. We find that interaction turnover was much larger across space than time, demonstrating that the spatial context leads to a significant rewiring of interactions. Our study shows significant spatial and temporal dynamics that we are currently far from understanding and that explain why predicting interactions is still a failed subject in ecology.

\section{Introduction}

In recent years, community-level analyses of plants and their pollinators have allowed us to understand a lot about how these communities are structured (Bascompte \& Jordano 2013), as well as have initial insights into how this structure affects some ecosystem functions (Magrach et al. 2021). However a major challenge continues to be predicting (both corroboratory and anticipatory) the structure of these communities.

Indeed, prediction is one of the main challenges faced by ecology today. In the case of interactions, different approaches have been followed to infer interactions from species composition through the use of trait-matching (Bartomeus et al. 2016), or machine-learning techniques (Strydom et al. 2021). But any of these approaches will fail to fully predict the full network of interactions, because although there are some emergent network properties that are highly predictable (e.g., connectance (MacDonald et al. 2020)), and trait-matching plays an important role in determining interaction frequencies, some interactions follow neutral processes which result from random encounters between individuals based on their abundance (Vázquez et al. 2009). At present, we do not know the proportion of links that are a consequence of these neutral forces, and whether this proportion changes across different habitat types. In addition, there are three main issues that are hampering our advances in the predictive front, (1) most information we have on plant-pollinator interactions comes from very different habitat types, such that we are currently unable to understand how generalizable our results are, (2) sampling completeness is low across most studies, but the reasons explaining these low coverage values are currently unknown, and related to this (3) we tend to assume that the co-existence of two species that have interacted in the past, means they will interact (Poisot et al. 2014), but this is not always the case, and we currently do not know why. 
Up to date, researchers have done multiple efforts across different habitat types to try to understand the patterns and processes that lead to the observed plant and pollinator community structures. These include efforts across arctic heathlands (Olesen et al. 2008a), subalpine meadows (CaraDonna et al. 2017), agricultural landscapes (Ponisio et al. 2017), xeric shrublands (Chacoff et al. 2017, ChávezGonzález et al. 2020), or tropical ecosystems (Kaiser-Bunbury et al. 2014). Review efforts have then tried to find general patterns using insights from these studies (CaraDonna et al. 2020b, Trøjelsgaard \& Olesen 2016), however currently it is difficult to disentangle the effects of the different habitat types from potential sampling artefacts, since each of these different studies have been done following different methodologies and sampling procedures. There are few studies that have accomplished to collect data in the exact same way across different habitat types in order to try to understand the generality of some of the findings, while eliminating the noise due to sampling differences.

Furthermore, sampling artefacts can also affect the network structure and the composition of the interactions observed (Vázquez et al. 2009). In many instances, sampling efforts are not sufficient to provide robust estimates of species and link presence and abundances, leading to small sampling completeness values (Chacoff et al. 2011). These low coverage values can be due to several causes, for example the type of sampling conducted (e.g., transects vs pan traps), the type of interaction sampled, the delimitation of the study area, the establishment of boundaries around the study system or the turnover in species and links. Moreover, interactions following neutral processes will likely reduce sampling coverage, but can we improve completeness if we are able to detect and remove these neutral interactions from our overall datasets?

In the past decades an increasing body of literature has highlighted the need to consider both temporal and spatial dynamics when studying plant and pollinator communities and their interactions (Burkle \& Alarcon 2011, CaraDonna et al. 2020a, Schwarz et al. 2021, Schwarz et al. 2020, Trøjelsgaard \& Olesen 2016). Through this spatial and temporally-explicit approaches we have become aware that the different phenology of plant and pollinator species leads to temporal changes in the composition of plant and pollinator communities, which leads to shifts in interaction composition, and consequently to changes in community structure (CaraDonna et al. 2017), ultimately affecting ecosystem functioning (Magrach et al. 2020). We have also learned that plant and pollinator species vary in their distribution in space, which leads to spatial changes in the composition of the community and thus to differences in the composition of interactions (Olesen \& Jordano 2002, Vázquez et al. 2009). But, changes in species composition are not the only reason why interaction composition can shift. Species do not necessarily interact in the same way with previous partners across space or time (Poisot et al. 2014). Interaction rewiring, or switching of partners, can also occur, for example through intraspecific variability in partner selection. At small spatial scales, these differences might be a consequence of differences in microclimate, radiation or soil type (Reverté et al. 2019), while at larger spatial scales other variables like land use or elevation could be driving these differences.

However, most approaches have either focused on spatial or temporal dynamics, and few have simultaneously considered both (Trøjelsgaard \& Olesen 2016). But only by considering them both at the 
same time will we be able to understand the mechanisms underlying community structure which will allow us to try to predict interactions and their responses to different perturbations.

Here, we use data collected following the same methodology within two different habitat types (mountain grasslands and the understory of Mediterranean woodlands) across two consecutive years. Data was collected at 5 locations in space and 8-9 times during the flowering season within each habitat type. Our aim is to use such a well-resolved spatial and temporal dataset to (i) assess the proportion of total interactions that consistently occur across different years, which would be those we could aim at predicting, (ii) understand whether spatial or temporal dynamics determine differences in community structure, and (iii) whether the patterns observed can be generalized across different habitat types.

\section{Methods}

\section{Study areas}

We collected data at two different areas in Spain: within the area of influence of Doñana National Park in SW Spain and at Gorbea Natural Park, located in N Spain (Fig. S1). The area of Doñana features a Mediterranean climate, with warm dry summers and cool humid winters. Annual precipitation is $500 \mathrm{~mm}$ and mean temperatures during the study period range from 12.5 to $22.5^{\circ} \mathrm{C}$ (Pizarro et al. 2021). Surveys were conducted within fragmented woodlands of stone pines (Pinus pinea) that host a rich understory of flowering shrubs and annual plants (Aparicio 2007). In turn, surveys in Gorbea were conducted within an area characterized by the influence of the Atlantic Ocean, with mild winters and summers and high annual precipitation values, $\sim 1,300 \mathrm{~mm}$. Here, mean temperatures during the study period range from 10 to $17.5^{\circ} \mathrm{C}$ (Pizarro et al. 2021). Vegetation in the area is composed of beech forests, in conjunction with abundant coniferous plantations. There is also a substantial presence of shrubland and meadows, together with dispersed outrocks, present mainly in the steepest areas of the mountain (Albizu et al. 2002).

Surveys were conducted during two consecutive years, 2020 and 2021. Within each area we selected five sites within similar elevations (ranging from 50 to $150 \mathrm{~m}$ a.s.l. in the case of Doñana and between 800 and 900 m.a.s.l in the case of Gorbea), as well as within similar habitat and soil types, reducing potential confounding factors. Similarity in plant composition between sites was $0.41 \pm 0.04$ in both areas and across both study years (plant mean Sørensen beta-diversity). The average distance between sites ranged from 3.2 to 24.2 in the case of Doñana and from 1.3 to $4.6 \mathrm{~km}$ in Gorbea. In Doñana, one of the sites had to substituted in 2021 due to impossibility of access in the second year of sampling and it was changed to a nearby very similar area.

Within each site, we established one 100-m x $2 \mathrm{~m}$ transect. Transects were visited every week (period), when weather conditions allowed, and surveyed three times during each visit, one in the early morning (9.30-10.30), one in the middle of the day (13.30-14.30) and one at the end of the day (16.30-17.30), in order to obtain a complete picture of the community of plants and pollinators present throughout the day. 
During the whole flowering season (from February to May in the case of Doñana and from March to July in the case of Gorbea), we were able to sample each of the sites between 6 and 9 times depending on the year. Sampling was interrupted for two weeks in 2020 due to the lockdowns derived from the COVID-19 pandemic. The objective was to sample all five sites for one full day each during the week, and then repeat the sampling again the following week in a randomized order, such that each week represented a period with a difference of 4 days in the sampling of each site. This was however not always possible due to lockdowns, restrictions and bad weather, and therefore in a small number of occasions, a period covers a maximum of 10 days difference between the sampling of the first site and that of the last.

At each census, along each transect we identified all plant species and recorded all the floral visitors that landed on their flowers and touched the plant's reproductive parts. Only floral visitors (from now on referred to as pollinators) that could not be identified in the field were captured, stored and identified in the laboratory by an expert entomologist (see acknowledgements). In addition, for each period, we recorded the number of flowers produced by each plant species present. All surveys were done under similar weather conditions, avoiding windy or rainy days. Total sampling time was 435 hours for both areas and years.

\section{Sampling completeness}

We assessed sampling completeness for plant and pollinator species as well as for plant-pollinator links using the Chao1 estimator of asymptotic species richness for abundance data (Chao 1984). To this end, we estimated the richness of plant, pollinator species and plant-pollinator links accumulated as sampling effort increased up to $100 \%$ sampling coverage using package iNEXT (Hsieh et al. 2016). In order to assess whether different sampling breadths (both temporal and spatial) could affect our sampling completeness estimates, we estimated species and link accumulation curves grouping data in four different ways. First, we estimated sampling completeness for the overall data obtained for each study area, pooling together all data for all sites and periods. Second, we estimated sampling completeness per period, i.e., by pooling together all the data across the five sites sampled per area for each period, but obtaining different sampling completeness values for each period of time. Third, we estimated sampling completeness per site, following a similar approach but this time pooling together all the data obtained for each of the five sites per study area across all the sampling periods. Finally, fourth, we estimated sampling completeness per site and period, i.e., for each of the individual censuses carried out for each site.

We calculated the proportion of interactions that were common across both study years for each study area, i.e., all the interactions that we were able to record in the two study years. To this end, we used the subset of data where pollinator specimens were identified to species level, i.e., we removed species identified solely to genus or other higher taxonomic order, which represents $85 \%$ of the total sampled specimens. We then further subsetted the data to include only the interactions between plants and pollinators that were observed both study years ( $52 \%$ of all data collected across both years and study areas) and calculated sampling completeness for the four different grouping ways as before. 


\section{Species and interaction turnover}

We calculated spatial and temporal turnover in plant and pollinator species as well as in plant-pollinator links. In the case of species, we calculated temporal turnover for each site as the total number of species found within that site over the entire study period minus the mean number of species per period for that site. Similarly, we calculated spatial turnover as the total number of species across the entire area minus the mean number of species per site (Tylianakis et al. 2005).

In the case of plant-pollinator links we calculated turnover in interaction composition across space and time involving species of plants and pollinators that were present across both sampling periods or sites being compared, i.e., we focused on the betaOS component of the turnover decomposition suggested by Poisot and colleagues (Poisot et al. 2012), i.e., that which refers to rewiring in species interactions. This metric takes values of 0 when interaction composition of the shared species is exactly the same and values of 1 when interaction composition is completely different.

As previously, we ran this analysis for the full dataset as well as for the subset of data that include only plant-pollinator links that are present in both study years for each area.

\section{Plant-pollinator network analysis}

To evaluate differences in community structure and species roles across space and time, we constructed plant-pollinator interaction networks by pooling together the data obtained for the three rounds of sampling per sampling day. We thus obtained one quantitative interaction network per site and period for each study area and year, representing the frequency of visits by different pollinator species to different plant species. For each network, we extracted a series of relevant metrics at the species and at the community level. Specifically, we focused on two metrics, complementary specialization ( $\left.\mathrm{H} 2^{\prime}\right)$ and the degree of interaction specialization ( $d^{\prime}$ ) of plant and pollinator species, which give an idea of the degree of specialization at the level of the whole community and at the species level respectively (Blüthgen et al. 2006). We calculated these metrics for the full dataset as well as for the subset of data only including interactions present across both years.

\section{Data analysis}

We evaluated whether there were differences in sampling completeness as a function of the type of pooling done with the data, i.e., whether there were differences in sampling coverage if this was calculated pooling all data together (overall), if data was pooled across sites, across periods or across sites and periods (i.e., for each census). To this end, we ran a general linear mixed model (LMM), with sampling completeness for either plants, pollinators or links as response variable, pooling type as independent variable and study area and year as random effects. We further evaluated pairwise differences across the different levels of pooling types by means of post hoc Tukey tests. We repeated the same kind of analysis using sampling completeness measured for the reduced dataset in which only interactions found both years were included. 
Following this same logic, we evaluated whether species and interaction turnover was larger across space or across time using LMMs with turnover as the response variable, turnover type (spatial or temporal) and the total number of species or interactions as independent variables and study area and year as random effects. As before, we ran this analysis using the full dataset, as well as the subset of data that included only interactions that are present across both study years.

Finally, we evaluated whether interaction network metrics varied across space and time by means of LMMs. We fitted two complementary LMMs for each network metric, which included the network metric $\left(\mathrm{H} 2^{\prime}\right.$ or $\mathrm{d}^{\prime}$ ) as response variable and the number of plant and pollinator species (which were not significantly correlated Spearman correlation $=0.43$ ). The first model, testing spatial differences in network structure also included site as independent variable, and period nested within year and study area as random factors. In turn, the second model included period as independent variable and site nested within year and study area as random factors. All models were fitted using a binomial distribution. We ran both models and selected the best performing using an information criterion approach based on the lowest AIC value. In the case of the species-level specialization, we included species as a random factor as well. As in the previous analyses, we then repeated this analysis but using the subset of data that included interactions present both years.

\section{Results}

We recorded a total of 56 flowering plant species in Doñana across both study years, and 82 in Gorbea. Out of these, $78.57 \%$ of the species received at least one visit by floral visitors in Doñana, while $65.85 \%$ of plant species present in Gorbea during the study period received visits by floral visitors.

We recorded a total of 156 and 130 species or morphospecies of flower visitors in Doñana and Gorbea respectively across both study years, and 44 and 54 species of plants respectively. Our survey included a total of 2,623 and 2,646 flower visits in Doñana and Gorbea respectively and 447 and 438 unique combinations of plants and flower visitors respectively. In Doñana, the most represented order of flower visitors was Hymenoptera, involved in $68.66 \%$ of the interactions observed. Specifically, Apidae represented the most common family within the order, representing $48.11 \%$ of the interactions and interactions involving Apis mellifera, the managed European honeybee, represented $41.67 \%$ of all interactions. Following Hymenoptera, the next most common order was Coleoptera representing $10.79 \%$ of the interactions. In turn, Diptera, and specifically the family Syrphidae, represented $10.26 \%$ of the interactions and Lepidoptera 1.75\%. In Gorbea, the most abundant group were Diptera, and specifically the family Syrphidae which accounted for $55.06 \%$ of the interactions, followed by Hymenoptera, representing $25.74 \%$, and specifically the family Apidae representing $17.23 \%$, but in this case Apis mellifera represented $9.33 \%$ of the interactions and different species in the Bombus genus $7.11 \%$.

A closer inspection of the phenology of the main different taxonomic groups across both study sites, shows differences in the importance (relative abundance) of each taxonoomic group at each of the two study sites and across both years (Fig. 1). For example, while the families Apidae and Syrphidae have a 
similar constant relative abundance through time in Gorbea, these two groups show contrasting temporal distributions in Doñana, with Syrphidae flower visitors dominating more at the beginning of the flowering season and then giving way to members of the group Apidae later on, mainly represented by the species Apis mellifera.

\section{Sampling completeness}

Our analysis of sampling completeness across our two areas sampled shows with our sampling efforts we were able to obtain high sampling coverage for both plant and pollinator communities in both study years ( $81 \%$ and $79 \%$ of pollinator species detected in 2020 and 2021 in the case of Doñana and $61 \%$ and $52 \%$ in the case of Gorbea, and $95 \%$ and $88 \%$ of plant species respectively for 2020 and 2021 in the case of Doñana and $92 \%$ and $94 \%$ in the case of Gorbea). In the case of plant-pollinator links, sampling completeness is lower in both study areas $(60 \%$ and $60 \%$ respectively for 2020 and 2021 in the case of Doñana and $43 \%$ and $51 \%$ in the case of Gorbea, Fig. 2).

These values are different when different sampling breadths are considered when calculating sampling completeness values. For example, if sampling completeness is calculated at the level of each of the independent surveys, i.e., for each site at each particular period (sampling week), we obtain highly variable coverage values ranging from 21.62 to $97.46 \%$ in the case of pollinators in Doñana and from 28.24 to $100 \%$ in the case of Gorbea. Similarly, in the case of plant species, sampling completeness is more variable in the case in which independent surveys are considered as sampling breadth, with between 51.61 to $100 \%$ of plant species being identified in the case of Doñana and between 27.75 and $100 \%$ in the case of Gorbea. In the case of links, these values ranged from 15.39 to $85.85 \%$ in the case of Doñana and between 11.3 and $100 \%$ in Gorbea.

An inspection of interactions that are common across both study years for both study sites, shows that $32.66 \%$ and $57.51 \%$ of the interactions observed in Doñana in 2020 and 2021 are unique to that year, i.e., they are not observed the other year. In turn, in Gorbea these proportions stand at $48.83 \%$ and $34.45 \%$ respectively for 2020 and 2021. In turn, pollinator species that are uniquely found in one of the study years represent $27.19 \%$ and $55.26 \%$ of all pollinator species in 2020 and 2021 respectively in the case of Doñana, and $35.62 \%$ and $27.4 \%$ in the case of Gorbea. For plant species, values of species uniquely found one of the study years stand at $33.33 \%$ and $28.57 \%$ in 2020 and 2021 for Doñana and $26.92 \%$ and $17.31 \%$ for Gorbea.

Subsetting our data to include only those interactions that are common across years ( $52.3 \%$ of the total interactions observed across both study areas and years), shows significant improvements in sampling completeness values for plants, pollinators and links at both sites, but particularly in the case of Doñana where link sampling completeness reaches $83 \%$ and $84 \%$ for 2020 and 2021 respectively and $66 \%$ and $69 \%$ in the case of Gorbea (Fig. S1).

Our statistical analysis of the sampling completeness estimates reveals there are no differences in coverage values of plant and pollinator species as well as plant-pollinator links between pooling types 
when the full dataset is used (Table 1). However, when we use the subset data, that includes only plantpollinator links present across both study years, we do find significant differences between pooling types for plant species, pollinator species as well as for plant-pollinator links. Specifically, we find major differences between coverage values calculated when data are pooled per site and period (i.e., when data obtained for one sampling day are used to calculate coverage) and those obtained for other pooling methods (see Table 2 for specific pairwise comparisons).

Table 1. Results of post hoc Tukey analyses evaluating pairwise differences between pooling types in sampling completeness values when using the full dataset for A) plant species, B) pollinator species and C) plant-pollinator links. Bold letters indicate pairwise combinations that are significantly different.

\begin{tabular}{llllll} 
A) Pooling type & estimate & SE & df & t.ratio & p.value \\
\hline overall - per period & 0.14 & 0.09 & 162 & 1.63 & 0.36 \\
\hline overall - per site & 0.12 & 0.09 & 162 & 1.33 & 0.55 \\
\hline overall - per site and period & 0.08 & 0.08 & 162 & 0.98 & 0.76 \\
\hline per period - per site & -0.02 & 0.05 & 162 & -0.51 & 0.96 \\
\hline per period - per site and period & -0.06 & 0.04 & 162 & -1.72 & 0.32 \\
\hline per site - per site and period & -0.04 & 0.04 & 162 & -0.96 & 0.77
\end{tabular}

\begin{tabular}{llllll} 
B) Pooling type & estimate & SE & df & t.ratio & p.value \\
\hline overall - per period & 0.10 & 0.11 & 162 & 0.89 & 0.81 \\
\hline overall - per site & 0.07 & 0.11 & 162 & 0.68 & 0.91 \\
\hline overall - per site and period & 0.09 & 0.10 & 162 & 0.87 & 0.82 \\
\hline per period - per site & -0.02 & 0.06 & 162 & -0.36 & 0.98 \\
\hline per period - per site and period & -0.01 & 0.04 & 162 & -0.17 & 1.00 \\
\hline per site - per site and period & 0.01 & 0.05 & 162 & 0.29 & 0.99
\end{tabular}




\begin{tabular}{llllll} 
C) Pooling type & estimate & SE & df & t.ratio & p.value \\
\hline overall - per period & 0.05 & 0.11 & 162 & 0.42 & 0.98 \\
\hline overall - per site & 0.09 & 0.11 & 162 & 0.79 & 0.86 \\
\hline overall - per site and period & 0.04 & 0.10 & 162 & 0.37 & 0.98 \\
\hline per period - per site & 0.04 & 0.06 & 162 & 0.69 & 0.90 \\
\hline per period - per site and period & -0.01 & 0.04 & 162 & -0.17 & 1.00 \\
\hline per site - per site and period & -0.05 & 0.05 & 162 & -1.02 & 0.74
\end{tabular}

Table 2. Results of post hoc Tukey analyses evaluating pairwise differences between pooling types in sampling completeness values when using subset of data that includes interactions that are present across both study years for A) plant species, B) pollinator species and C) plant-pollinator links. Bold letters indicate pairwise combinations that are significantly different.

\begin{tabular}{llllll} 
A) Pooling type & estimate & SE & df & t.ratio & p.value \\
\hline overall - per period & 0.05 & 0.08 & 152 & 0.72 & 0.89 \\
\hline overall - per site & -0.02 & 0.08 & 152 & -0.32 & 0.99 \\
\hline overall - per site and period & -0.08 & 0.07 & 152 & -1.06 & 0.71 \\
\hline per period - per site & -0.08 & 0.04 & 152 & -1.87 & 0.25 \\
\hline per period - per site and period & -0.13 & $\mathbf{0 . 0 3}$ & 152 & -4.13 & 0.00 \\
\hline per site - per site and period & -0.05 & 0.03 & 152 & -1.50 & 0.44
\end{tabular}

\begin{tabular}{llllll} 
B) Pooling type & estimate & SE & df & t.ratio & p.value \\
\hline overall - per period & -0.09 & 0.09 & 152 & -1.01 & 0.74 \\
\hline overall - per site & -0.04 & 0.09 & 152 & -0.44 & 0.97 \\
\hline overall - per site and period & -0.14 & 0.09 & 152 & -1.63 & 0.36 \\
\hline per period - per site & 0.05 & 0.05 & 152 & 1.02 & 0.74 \\
\hline per period - per site and period & -0.05 & 0.04 & 152 & -1.27 & 0.59 \\
\hline per site - per site and period & $\mathbf{- 0 . 1 0}$ & $\mathbf{0 . 0 4}$ & $\mathbf{1 5 2}$ & $\mathbf{- 2 . 4 4}$ & $\mathbf{0 . 0 4}$
\end{tabular}




\begin{tabular}{llllll} 
C) Pooling type & estimate & SE & df & t.ratio & p.value \\
\hline overall - per period & 0.06 & 0.11 & 152 & 0.57 & 0.94 \\
\hline overall - per site & 0.09 & 0.11 & 152 & 0.80 & 0.85 \\
\hline overall - per site and period & -0.04 & 0.10 & 152 & -0.36 & 0.98 \\
\hline per period - per site & 0.03 & 0.06 & 152 & 0.43 & 0.97 \\
\hline per period - per site and period & -0.10 & 0.05 & 152 & -2.19 & 0.13 \\
\hline per site - per site and period & $\mathbf{- 0 . 1 3}$ & $\mathbf{0 . 0 5}$ & $\mathbf{1 5 2}$ & $\mathbf{- 2 . 5 6}$ & $\mathbf{0 . 0 4}$
\end{tabular}

\section{Turnover}

Our analysis of spatial and temporal turnover in plant and pollinator species as well as plant-pollinator links, reveals different patterns for each one. Plant species show small values of turnover both through space and time at both study areas (Table 3a, Fig. 3). In turn, pollinator species show in general much larger values of temporal than spatial turnover (Fig. 3, Table 3b). The converse is true for turnover in links involving species that are present across periods of time or across sites, which show greatest spatial turnover values, meaning that despite there being the same species of plants and pollinators located at a given moment across the different surveyed sites, these species form different interacting pairs at each site (Fig. 3, Table 3c).

Table 3. Results of LMM analyzing differences between turnover types (spatial vs temporal) in A) plant species, B) pollinator species and C) plant-pollinator links. Table shows estimates and lower and upper confidence interval values. Bold letters indicate variables whose confidence intervals do not overlap 0.

\begin{tabular}{llllll} 
A) & Estimate & Std. Error & t value & $2.5 \%$ & $97.5 \%$ \\
\hline Intercept) & 2.52 & 1.03 & 2.44 & 0.52 & 4.52 \\
\hline Turnover type & 0.45 & 0.73 & 0.62 & -0.96 & 1.86 \\
\hline Species richness & $\mathbf{1 . 2 3}$ & $\mathbf{0 . 1 9}$ & $\mathbf{6 . 6 2}$ & $\mathbf{0 . 8 7}$ & $\mathbf{1 . 5 9}$
\end{tabular}

\begin{tabular}{llllll} 
B) & Estimate & Std. Error & t value & $2.5 \%$ & $97.5 \%$ \\
\hline (Intercept) & -0.39 & 2.62 & -0.15 & -5.33 & 4.44 \\
\hline Turnover type & $\mathbf{8 . 3 1}$ & $\mathbf{1 . 3 3}$ & $\mathbf{6 . 2 3}$ & $\mathbf{5 . 7 0}$ & 10.93 \\
\hline Species richness & $\mathbf{2 . 0 4}$ & $\mathbf{0 . 2 5}$ & $\mathbf{8 . 2 6}$ & $\mathbf{1 . 5 7}$ & $\mathbf{2 . 5 5}$
\end{tabular}




\begin{tabular}{llllll} 
C) & Estimate & Std. Error & t value & $2.5 \%$ & $97.5 \%$ \\
\hline Intercept) & 0.11 & 0.03 & 3.60 & 0.05 & 0.17 \\
\hline Turnover type & $-\mathbf{0 . 0 6}$ & $\mathbf{0 . 0 2}$ & $-\mathbf{3 . 5 0}$ & -0.09 & -0.03 \\
\hline Interaction richness & $\mathbf{0 . 0 0}$ & $\mathbf{0 . 0 0}$ & $\mathbf{3 . 5 5}$ & $\mathbf{0 . 0 0}$ & $\mathbf{0 . 0 0}$
\end{tabular}

As before, reducing our sample to include only plant-pollinator interactions that are recorded in both study years at each of the surveyed areas shows changes in the turnover of species and links (Fig. S2). While both spatial and temporal turnover decreases substantially for plant and pollinator species across both study areas, reducing the sample only to links common across both years still shows larger values of spatial than temporal turnover in link composition.

Table 4. Results of LMM analyzing differences between turnover $i$ ? types (spatial vs temporal) in A) plant species, B) pollinator species and C) plant-pollinator links. Data used is the subset of data including interactions that are present across both study years for each area. Table shows estimates and lower and upper confidence interval values. Bold letters indicate variables whose confidence intervals do not overlap 0 .

\begin{tabular}{llllll} 
A) & Estimate & Std. Error & $\mathrm{t}$ value & $2.5 \%$ & $97.5 \%$ \\
\hline (Intercept) & -0.21 & 0.62 & -0.34 & -1.45 & 1.01 \\
\hline Turnover type & 0.20 & 0.41 & 0.50 & -0.59 & 1.00 \\
\hline Species richness & 1.54 & 0.21 & 7.39 & 1.12 & 1.94
\end{tabular}

\begin{tabular}{llllll} 
B) & Estimate & Std. Error & t value & $2.5 \%$ & $97.5 \%$ \\
\hline Intercept) & 0.55 & 0.73 & 0.75 & -0.86 & 1.95 \\
\hline Turnover type & $\mathbf{1 . 2 0}$ & $\mathbf{0 . 4 6}$ & $\mathbf{2 . 6 1}$ & $\mathbf{0 . 3 1}$ & $\mathbf{2 . 0 9}$ \\
\hline Species richness & $\mathbf{0 . 9 3}$ & $\mathbf{0 . 2 3}$ & $\mathbf{4 . 0 9}$ & $\mathbf{0 . 4 9}$ & $\mathbf{1 . 3 7}$
\end{tabular}

\begin{tabular}{llllll} 
C) & Estimate & Std. Error & $\mathrm{t}$ value & $2.5 \%$ & $97.5 \%$ \\
(Intercept) & 0.04 & 0.03 & 1.23 & -0.02 & 0.10 \\
\hline Turnover type & -0.06 & 0.02 & -3.33 & -0.09 & -0.02 \\
\hline Interaction richness & 0.01 & 0.00 & 3.36 & 0.00 & 0.01
\end{tabular}


For every model, we found that the best model selected (i.e., that with the lowest AIC) was the model that accounted for spatial differences in network metrics. In the case of complementary specialization, we however found that none of the variables included in the model successfully explained any of the variability (Table 5, Fig. 4). In turn, in the case of species-level specialization, we found significant differences across sites and differences that could be partially explained by differences in plant and pollinator species richness (Table 5, Fig. 4). Specifically, we found specialization decreased with increasing pollinator richness and increased with plant richness.

Table 5. Results of LMM analyzing spatial differences in A) complementary specialization ( $\left.\mathrm{H} 2^{\prime}\right)$ at the community level and B) species-level specialization (d'). Table shows Type II Wald chisquare tests. Bold letters indicate variables whose confidence intervals do not overlap 0.

\begin{tabular}{llll} 
A) & Chisq & Df & $\operatorname{Pr}(>$ Chisq $)$ \\
\hline Site & 14.94 & 9 & 0.09 \\
\hline Plant sps & 0.57 & 1 & 0.45 \\
\hline Pollinator sps & 0.02 & 1 & 0.88
\end{tabular}

\begin{tabular}{llll} 
B) & Chisq & Df & $\operatorname{Pr}(>$ Chisq $)$ \\
\hline Site & 30.49 & 9 & 0.00 \\
\hline Plant sps & 6.42 & 1 & 0.01 \\
\hline Pollinator sps & 8.31 & 1 & 0.00
\end{tabular}

The analysis using the subset of data that included only interactions present in both study years, shows similar non-significant differences for complementary specialization, and changes in species-level specialization across surveyed sites (Table S1).

\section{Discussion}

Our results show strong spatio-temporal variability in plant-pollinator interactions driven by differences in pollinator specialization levels as a consequence of changes in plant and pollinator species richness. This variability at small spatial scales has already been observed within synthesis works (e.g., (Trøjelsgaard \& Olesen 2016)), but to the best of our knowledge this is the first attempt at simultaneously evaluating temporal and spatial variabilities to infer their different effects. Interestingly, we find that although temporal variability is important, and in particular drives changes in the composition of pollinator species communities, spatial differences in interaction composition seem to override temporal 
differences. In this case, these differences are not a consequence of changes in the overlap of species, since we focus on the turnover of shared species across space or time, but rather are a consequence of changing interacting patterns between shared species due to changes in the overall composition of plants and pollinators. For example, we find that species level specialization changes through space, increasing with pollinator species richness and decreasing with plant species richness. It therefore seems that in the presence of direct competition pollinator species become more specialized, while they become more generalized when the availability of different plant resources increases.

We find that sampling completeness varied as a function of how data were pooled. Specifically, when spatial and temporal dynamics were not considered, i.e., when data were pooled for each site and period, sampling completeness greatly increased, giving a false sense of complete sampling coverage, when in reality such a reduced sampling size is omitting differences in species and link composition across space and time. These results are important because in many cases, the validity of network structure, summarised by a number of descriptive metrics, depends on how representative a sample is from the overall population. Neglecting to take into account spatial or temporal dynamics could thus be affecting our understanding of many natural ecosystems.

Within our dataset, we find that while most plant species were consistently recorded across both study years, a significant proportion of the interactions and pollinator species were uniquely recorded during one of the study years. In the case of pollinator species, we find values of pollinator persistence across years ranging from 54 to $73 \%$. These values are intermediate compared to those found in other studies. For example, (Olesen et al. 2008b) found that $80 \%$ of the pollinator species present in an arctic heathland were recorded during two consecutive study years, (Dupont et al. 2009) found that this value decreased to $<25 \%$ of the total pollinator fauna, while in the case of (Petanidou et al. 2008) only $20.5 \%$ of pollinator species were detected in all of four study years. Interestingly, these results arise despite our large sampling efforts, spanning multiple locations and times, and including several surveys throughout the day to account for daily dynamics. This could mean that our sampling was still too low to uncover all existing species and interactions, or it could mean that some of these interactions follow neutral processes, and are a consequence of random encounters between plants and pollinators based on species abundances (Vázquez et al. 2009), which do not necessarily replicate every year as species abundances shift through time (e.g., bet hedging Danforth 1999).

A re-estimation of sampling completeness removing these interactions that were uniquely recorded during one study year greatly improves coverage values in the case of plant-pollinator links, but decreases the sampling completeness of pollinator species in the case of Gorbea. This shows that a significant portion of the pollinator species in this area were involved in unique interactions not recorded both years, which could be a consequence of pollinator species in this area having more of an "opportunistic" behavior, changing their plant preference as a consequence of changing plant species abundances. Indeed, Gorbea, located in a mountain area features much larger environmental differences throughout the day as well as between weeks, which probably means pollinators are more adaptive to changing conditions (Ploquin et al. 2013). Pollinator communities in this area are more plastic in their 
use of resources, and will adapt better to future perturbations, such as climate change, or maybe our findings show that they are already adapting to it, as mountain areas are one of the most impacted by changing climates (Inouye 2019).

Further, our results show that while the community of plant species is relatively constant across space and time, pollinator species turnover is greatest through time, i.e., the composition of pollinator species changes more for a particular site through time than across sites for a particular time period. In the case of plant-pollinator links occurring between shared plant and pollinator species, we find the contrary. In this case, turnover is largest across space, i.e., given the same species of plants and pollinators, these interact more similarly through time for a given location than they do across space for a given period. Reducing our dataset to only interactions common in both years, significantly reduces plant and pollinator species turnover, but retains similar values of interaction turnover, particularly spatial turnover, which means that variability in interaction composition amongst shared species through space is a prevalent trend across both study areas. Therefore, not only do we have a significant portion of interactions occurring only in one study year, but amongst those that occur across multiple years, there are strong spatial differences in the frequency in which they occur.

A closer inspection at community structure metrics, shows that it is also space that acts as the main driver of differences in both community and species-level specialization values. In the case of specieslevel specialization, part of the variability can be explained through spatial differences in plant and pollinator species richness, such that pollinator specialization increases in the face of increasing competition by other pollinator species and decreases with the increase in plant resources. This therefore answers, at least partially, the question posed by (Trøjelsgaard \& Olesen 2016), "why do some species interact one year but not the subsequent although they co-occur in both years?" Our results show that together with trait-matching and neutral processes driven by differences in species abundances (Vázquez et al. 2009), the composition of both interacting communities plays an important role in determining species interactions. This probably explains why predictive exercises have been somewhat successful in predicting macroscopic features of plant and pollinator communities, but no so much in predicting pairwise interactions (Vázquez et al. 2009)(Olito \& Fox 2014), which vary across space and time (Trøjelsgaard \& Olesen 2016).

In summary, our results show the importance of simultaneously considering spatial and temporal dynamics within natural communities. A step further should try to understand the mechanisms that drive these spatial and temporal differences and the biotic and abiotic factors that are driving them, and that will ultimately help us improve our predictions. These results are also important for conservation or restoration efforts, that should also consider the necessary micro-scale dynamics needed to maintain the stability and functioning of natural ecosystems.

\section{Declarations}

\section{Data accessibility}


All data will be deposited on an online repository (e.g., DataDryad) upon acceptance of the manuscript.

\section{Acknowledgements}

We thank Curro Molina for identifying most of the pollinator specimens collected at both study areas and Ignasi Bartomeus for comments on a previous version of the manuscript. Research for this project was funded through a project awarded by the Ministry of Science and Innovation in the framework of the "Proyectos de I+D Generación del Conocimiento" framework (PGC2018-098498-A-100). A.M. received funding from an Ikerbasque Research Fellowship. Research was also supported by the Spanish State Research Agency through Maria de Maeztu Excellence Unit accreditation (MDM-2017-0714) and the Basque Government BERC Programme.

Author Contributions: AM originally formulated the idea, AM, MA, PDL, CP and ER conducted fieldwork, AM performed statistical analyses, and wrote the first version of the manuscript, all authors contributed to revisions and improvements to the manuscript.

\section{References}

1. Albizu I, Besga G, Garbisu C, Mendarte S, Amezaga I, Mijangos I et al (2002) Comparación de la altura y unidades del medidor de placa como estimadores de la biomasa en los pastos de montaña del área de gorbeia (Bizkaia)

2. Aparicio A (2007) Descriptive analysis of the 'relictual' Mediterranean landscape in the Guadalquivir River valley (southern Spain): a baseline for scientific research and the development of conservation action plans. Biodivers Conserv 17:2219-2232

3. Bartomeus I, Gravel D, Tylianakis JM, Aizen MA, Dickie IA, Bernard-Verdier M (2016) A common framework for identifying linkage rules across different types of interactions. Funct Ecol 30:18941903

4. Bascompte J, Jordano P (2013) The structure of plant-animal mutualistic networks. Princeton University Press

5. Blüthgen N, Menzel F, Blüthgen N (2006) BMC Ecol 6:9

6. Burkle LA, Alarcon R (2011) The future of plant-pollinator diversity: Understanding interaction networks across time, space, and global change. Am J Bot 98:528-538

7. CaraDonna PJ, Burkle LA, Schwarz B, Resasco J, Knight TM, Benadi G et al (2020a) Seeing through the static: the temporal dimension of plantanimal mutualistic interactions. Ecol Lett 24:149-161

8. CaraDonna PJ, Burkle LA, Schwarz B, Resasco J, Knight TM, Benadi G et al (2020b) Seeing through the static: the temporal dimension of plantanimal mutualistic interactions. Ecol Lett 24:149-161

9. CaraDonna PJ, Petry WK, Brennan RM, Cunningham JL, Bronstein JL, Waser NM et al (2017) Interaction rewiring and the rapid turnover of plantpollinator networks. Ecol Lett 20:385-394

10. Chacoff NP, Resasco J, Vázquez DP (2017) Interaction frequency, network position, and the temporal persistence of interactions in a plantpollinator network. Ecology 99:21-28 
11. Chacoff NP, Vázquez DP, Lomáscolo SB, Stevani EL, Dorado J, Padrón B (2011) Evaluating sampling completeness in a desert plant-pollinator network. J Anim Ecol 81:190-200

12. Chao A (1984) Nonparametric estimation of the number of classes in a population. Scand J Stat $11: 265-270$

13. Chávez-González E, Vizentin-Bugoni J, Vázquez DP, MacGregor-Fors I, Dáttilo W, Ortiz-Pulido R (2020) Drivers of the structure of planthummingbird interaction networks at multiple temporal scales. Oecologia 193:913-924

14. Danforth BN (1999) Emergence dynamics and bet hedging in a desert bee, Perdita portalis. Proceedings of the Royal Society of London. Series B: Biological Sciences, 266, 1985-1994

15. Dupont YL, Padrón B, Olesen JM, Petanidou T (2009) Spatio-temporal variation in the structure of pollination networks. Oikos 118:1261-1269

16. Hsieh TC, Ma KH, Chao A (2016) iNEXT: an R package for rarefaction and extrapolation of species diversity ( $\mathrm{H}$ ill numbers). Methods in Ecology and Evolution, 7, 1451-1456

17. Inouye DW (2019) Effects of climate change on alpine plants and their pollinators. Ann N Y Acad Sci 1469:26-37

18. Kaiser-Bunbury CN, Vázquez DP, Stang M, Ghazoul J (2014) Determinants of the microstructure of plantpollinator networks. Ecology 95:3314-3324

19. MacDonald AAM, Banville F, Poisot T (2020) Revisiting the Links-Species Scaling Relationship in Food Webs. Patterns 1:100079

20. Magrach A, Lara C, Luna UM, Díaz-Infante S, Parker I (2020) Community-level reorganizations following migratory pollinator dynamics along a latitudinal gradient. Proceedings of the Royal Society B: Biological Sciences, 287, 20200649

21. Magrach A, Molina FP, Bartomeus I (2021) Niche complementarity among pollinators increases community-level plant reproductive success.Peer Community Journal,1

22. Olesen JM, Bascompte J, Elberling H, Jordano P (2008) Temporal dynamics in a pollination network. Ecology 89:1573-1582

23. Olesen JM, Jordano P (2002) Geographic patterns in plantpollinator mutualistic networks. Ecology 83:2416-2424

24. Olito C, Fox JW (2014) Species traits and abundances predict metrics of plant-pollinator network structure, but not pairwise interactions. Oikos 124:428-436

25. Petanidou T, Kallimanis AS, Tzanopoulos J, Sgardelis SP, Pantis JD (2008) Long-term observation of a pollination network: fluctuation in species and interactions, relative invariance of network structure and implications for estimates of specialization. Ecol Lett 11:564-575

26. Pizarro M, Hernangómez D, Fernández-Avilés G (2021) Climaemet:Climate AEMET tools

27. Ploquin EF, Herrera JM, Obeso JR (2013) Bumblebee community homogenization after uphill shifts in montane areas of northern Spain. Oecologia 173:1649-1660 
28. Poisot T, Canard E, Mouillot D, Mouquet N, Gravel D (2012) The dissimilarity of species interaction networks. Ecol Lett 15:1353-1361

29. Poisot T, Stouffer DB, Gravel D (2014) Beyond species: why ecological interaction networks vary through space and time. Oikos 124:243-251

30. Ponisio LC, Gaiarsa MP, Kremen C (2017) Opportunistic attachment assembles plantpollinator networks. Ecol Lett 20:1261-1272

31. Reverté S, Bosch J, Arnan X, Roslin T, Stefanescu C, Calleja JA et al (2019) Spatial variability in a plantpollinator community across a continuous habitat: high heterogeneity in the face of apparent uniformity. Ecography 42:1558-1568

32. Schwarz B, Dormann CF, Vázquez DP, Fründ J (2021) Within-day dynamics of plantpollinator networks are dominated by early flower closure: an experimental test of network plasticity. Oecologia 196:781-794

33. Schwarz B, Vázquez DP, CaraDonna PJ, Knight TM, Benadi G, Dormann CF et al (2020) Temporal scale-dependence of plantpollinator networks. Oikos 129:1289-1302

34. Strydom T, Catchen MD, Banville F, Caron D, Dansereau G, Desjardins-Proulx P et al (2021) A roadmap towards predicting species interaction networks (across space and time). Philosophical Trans Royal Soc B: Biol Sci 376:20210063

35. Trøjelsgaard K, Olesen JM (2016) Ecological networks in motion: micro- and macroscopic variability across scales. Funct Ecol 30:1926-1935

36. Tylianakis JM, Klein A-M, Tscharntke T (2005) Spatiotemporal variation in the diversity of hymenoptera across a tropical habitat gradient. Ecology 86:3296-3302

37. Vázquez DP, Blüthgen N, Cagnolo L, Chacoff NP (2009) Uniting pattern and process in plant animal mutualistic networks: a review. Ann Botany 103:1445-1457

\section{Figures}

\section{Figure 1}

Violin plots showing the relative importance of different taxonomic groups through time for each of the study sites surveyed in both study years.

\section{Figure 2}

Boxplots showing sampling completeness for a) plant species, b) pollinator species and c) plantpollinator links. Different colors represent different ways in which data can be analyzed, pink shows overall sampling completeness calculated using all data collected, purple completeness for each of the 
time periods grouping all sites together, orange shows completeness for each of the different sites separately grouping data for the different periods of time in which each site is sampled and blue shows completeness for each individual sampling event, i.e., for each site at each period.
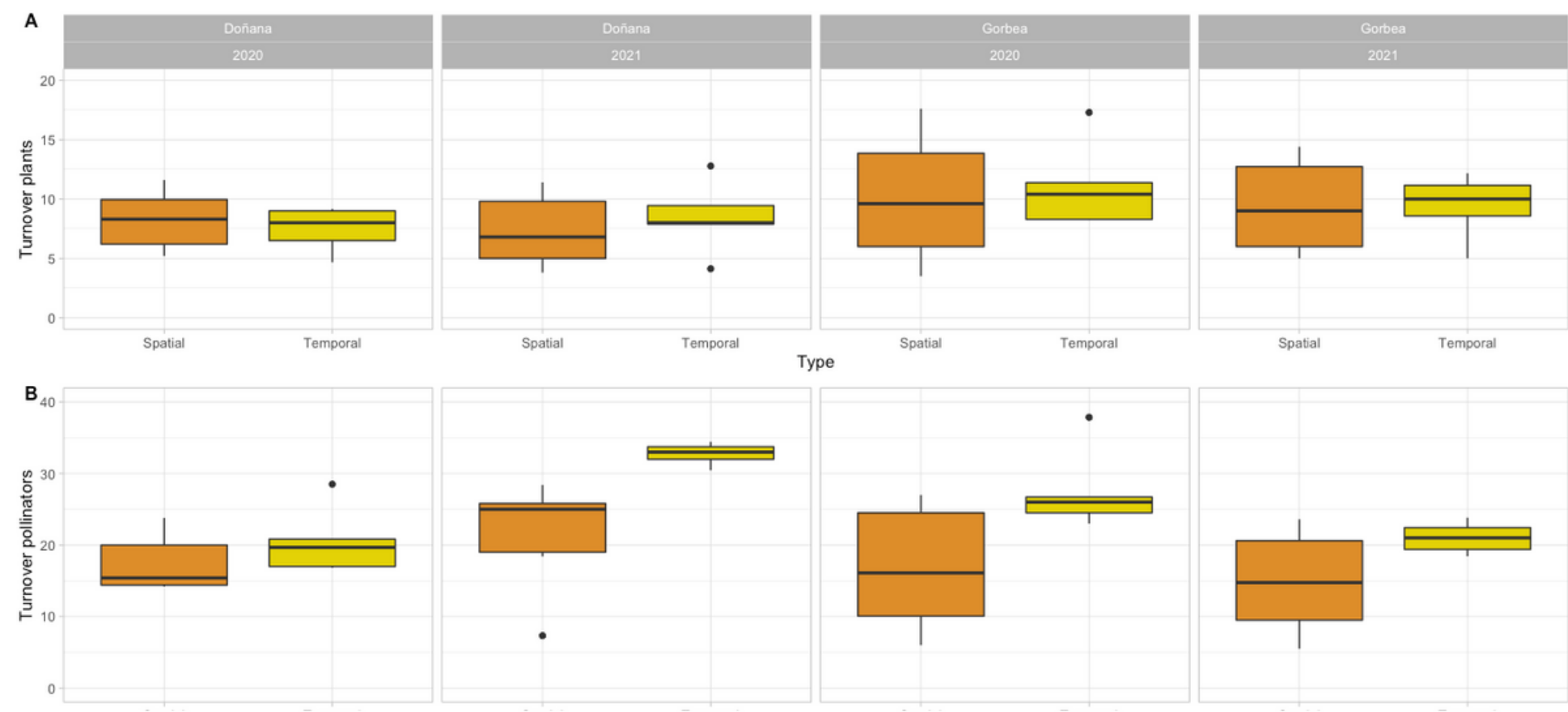

\section{Figure 3}

Boxplots showing spatial and temporal turnover for a) plant species, b) pollinator species and c) plantpollinator links including plant and pollinator species that are common across periods or sites.

\section{Figure 4}

A) Boxplot showing differences in complementary specialization across sampled sites in the two study areas and years. B)-C) Scatterplots showing effects of pollinator and plant species richness respectively on species-level specialization.

\section{Supplementary Files}


This is a list of supplementary files associated with this preprint. Click to download.

- Supplementarymaterial.docx 